\title{
Semantic Adaptation of Knowledge Representation Systems
}

\author{
Catarina Lucena $^{1,2}$, João Sarraipa ${ }^{1,2}$, and Ricardo Jardim-Gonçalves ${ }^{1,2}$ \\ ${ }^{1}$ Departamento de Engenharia Electrotécnica, Faculdade de Ciências e Tecnologia, FCT, \\ Universidade Nova de Lisboa, 2829-516 Caparica, Portugal \\ ${ }^{2}$ Centre of Technology and Systems, CTS, UNINOVA, 2829-516 Caparica, Portugal \\ $\{\mathrm{cml}, \mathrm{jfss}, \mathrm{rg}\}$ @uninova.pt
}

\begin{abstract}
Due to the worldwide diversity of enterprises, a high number of ontologies representing the same segment of reality which are not semantically coincident have appeared. To solve this problem, a possible solution is to use a reference ontology to be the intermediary in the communications between the community enterprises and to outside. Since semantic mappings between enterprise's ontologies are established, this solution allows each of the enterprises to keep internally its own ontology and semantics unchanged. However information systems are not static, thus established mappings become obsolete with time. This paper's presents a $\mathrm{PhD}$ research with the objective to identify a suitable approach that combines semantic mappings with user's feedback, providing an automatic learning to ontologies \& enabling autoadaptability and, consequently, dynamism to the information systems.
\end{abstract}

Keywords: Ontologies, Knowledge Maintenance, Adaptability, Ontology Learning.

\section{Introduction}

The World Wide Web is a vast and growing source of information and services which need to be shared by people and applications. Ontologies play a major role in supporting the information exchange and sharing by extending syntactic interoperability of the Web to semantic interoperability. However, since information systems are not static, ontologies cannot be thought as an achieved conceptualization of well-delimited and static domain. One of the important aspects in the evolution process is to guarantee the consistence of the ontology when changes occur. That requires a semantic adaptation of its represented knowledge.

Adaptability can be defined as the ability of a system to adapt itself efficiently and fast to changed circumstances. An adaptive system is therefore an open system that is able to fit its behaviour according to changes in its environment or in parts of the system itself [1]. One example is the Internet of Things (IoT) where smart interaction between objects that adapt to the current situation without any human involvement will become the next logical step to people stay connected anytime and anywhere. 


\section{Relationship to Internet of Things}

The Internet of Things (IoT) is a novel paradigm that is rapidly gaining ground in the scenario of modern wireless telecommunications. The basic idea of this concept is the pervasive presence around us of a variety of things or objects - such as RadioFrequency IDentification (RFID) tags, sensors, actuators, mobile phones, etc. - which, through unique addressing schemes, are able to interact with each other and cooperate with their neighbours to reach common goals [2].

Semantic oriented computing manifests its potential to cope with the challenging problems of heterogeneity and interoperability exposed by the large number of things with different characteristics. The application of semantic technology to the IoT domain will provide systems with the ability to better understand terms and concepts as data is transmitted from one system to another, while preserving the meaning of the content. There are many applications using semantic Web technologies in IoT research [3, 4], however, current work has mostly focused on IoT resources management while not on how to access and utilise information generated in IoT. It is also necessary to consider that a semantic model or ontology is not enough to our data be interoperable. There are/could be $n$ ontologies for a domain. In this sense is necessary to exist ontology mappings, reference ontologies and standardisation efforts. This PhD research can contribute to the Semantic Adaptation of Knowledge Representation through identification of a suitable approach that provides an automatic learning to ontologies \& enabling auto-adaptability and dynamism to the IoT information systems.

\section{Followed Research Method}

Research is a systematic process of collecting and analysing information to increase our understanding of the phenomenon under study. It is the function of the researcher to contribute to the understanding of the phenomenon and to communicate that understanding to others [5]. It is argued that the clear definition of a research strategy is a fundamental and necessary requirement for a sound empirical study [6]. The research method adopted by the authors (see Fig. 1) is an instantiation of the 7 classic phases scientific method [7]. The difference is that this has 8 phases, where, for each research question/hypothesis, the last phase aims the technological transfer to industry.

Is possible to see in Fig. 1that the first 5 steps will be repeated cyclically until prove/show the studied theory. By the last, the referred cycle must be carry out the number or times needed in order to mature all the small research question that compose the research overall objective, which in this case is to contribute to the "Semantic Adaptation of Knowledge Representation Systems". 


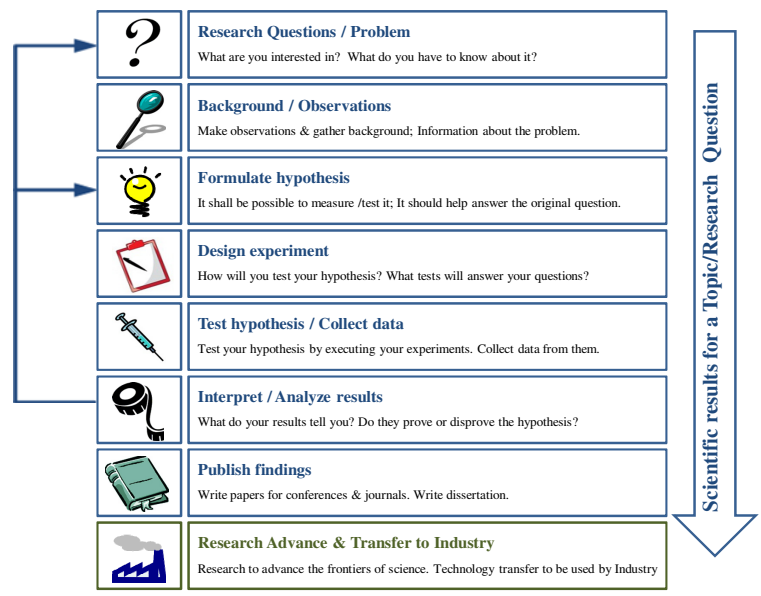

Fig. 1. Adopted Research Method

\subsection{Research Questions}

A research question is an inquiry that is asked for the purpose of gaining knowledge or useful information on a area of interest to which the authors is intended to participate and contribute for. Research questions are used to determine possibilities and gain valuable insight. Thus, the research questions to be addressed are: 1) How to improve ontology based systems to facilitate its intelligence increase? 2) How to enhance the knowledge acquisition from information system's external users? 3) How to improve the interoperability of software applications and information systems semantics?

Although to contribute to this it is needed to make a first background observation to identify some hypothesis in the resolution of the objective.

\subsection{Background Observations}

The ability to integrate and apply specialized knowledge of organizational members is fundamental to a firm's ability to create and sustain competitive advantage. This include the ability of organizations to be flexible and respond more quickly to changing market conditions, and the ability to be more innovative as well as improving decision making and productivity [8].

Knowledge Management is the process of capturing the collective expertise and intelligence in an organization, using it to promote innovation through continued organizational learning [9]. In additional, it has become a challenging activity for most competitive business organisations. There is growing recognition in the business community about the importance of knowledge as a critical resource for organisations $[10,11]$. Individuals and companies are obliged to focus on maintaining and enhancing their knowledge asset in order to innovate [11,12] and survive in the current competitive markets. 
It is now frequently assumed that knowledge is modelled and stored in structures called 'ontologies' which are defined as a formal and explicit specification of a shared conceptualization [13] and may be used as a unifying framework to facilitate knowledge sharing and interoperability between independently developed systems . Ontologies are computer implementations of human-like knowledge, for the purpose of describing domains of the world and sharing this knowledge between application programs (and also between people) [14]. Its recognised capacity to represent knowledge, to facilitate reasoning, use and exchange knowledge between systems contribute to increase the computational intelligence [15].

Due to the worldwide diversity of communities, a high number of ontologies representing the same segment of reality which are not semantically coincident have appeared. To solve this problem, a possible solution is to use a reference ontology to be the intermediary in the communications between the community enterprises and to outside. Since semantic mappings between enterprise's ontologies are established, this solution allows each of the enterprises to keep internally its own ontology and semantics unchanged. However information systems are not static, thus established mappings become obsolete with time.

Whether knowledge is stored in ontologies, prepositional knowledge based or simple databases, it must be maintained and kept up to date. There are many reasons for ontology changes: the continual evolution of the modelled domain, the refinement of the ontology conceptualization, the modification of the application by adding functionalities according to new end-user requirements and the reuse of the ontology for others tasks or applications. To take into account all these evolving aspects, ontologies have to be adapted to change requirements [16] in a formal dynamic Knowledge Maintenance (KMa) establishment. KMa is focused on the Knowledge Base improvement to actively be updated, monitored accordingly to the knowledge evolution of its related domain [17].

\subsection{Hypothesis}

The hypotheses to which the authors will follow to execute experiments are the following: 1) A proper interoperability system based on knowledge representation and reasoning is able to be adapted based on external feedback, facilitating the semantic adaptability on future enterprise systems 2) An ontology based framework integrated with proper operational research methods would facilitate the knowledge acquisition from user's feedback and would increase its ability to KMa 3) The next generation of intelligent systems to assist on interoperability of software applications and information systems needs the support of machine learning and operational research methods.

\section{Design Experiment}

This chapter presents a preliminary study to better understand the authors' research area, which has been used to properly design the experiments. There are introduced some statements about Artificial Intelligence (AI) and its parallelism to neuroscience addressing how human brain deals with semantic memories and learning. Then, 
human based learning techniques and Ontology Learning (OL) are introduced since together with some machine learning techniques could be used to facilitate knowledge systems maintenance.

Crucial systems to understand are those involved in memory, but in addition, learning mechanisms are at the heart of how the brain processed information. [18] states that is by modifying the synaptic connection strengths (or weights) between neurons that useful neuronal information processors for most brain functions, including perception, emotion, motivation, and motor function, are built. One example is the study made by Patterson in [19]. This study used basic emotions as a facilitator for learning. Thus, emotions were defined in this case, due to the animal's use, as states elicited by rewards and punishments. A reward is anything for which an animal will work. A punisher is anything an animal will work to escape or avoid. Rewards and punishments can be more formally defined as instrumental reinforcers, i.e. stimuli or events which, if their occurrence, termination, or omission is made contingent upon the making of a response, alter the probability of the future emission of that response.

Following the same idea, artificial intelligent semantic relatedness techniques/ methods can also be considered able to facilitate semantic adaptation ability to its connected system, inspired on brain learning. Since the main idea is to find a system able to learn, but also able to represent and manage complex inputs as concepts like humans do, one possible solution is to use OL. This refers to extracting ontological elements (conceptual knowledge) from input and build ontology from them [20]. Ontology learning can be defined as the set of methods and techniques used for building, semi-automatically or automatically, ontology from scratch, enriching, or adapting an existing ontology using several sources [21]. Compared with manually crafting ontologies, ontology learning is able to not only discover ontological knowledge at a large scale and faster pace, but also mitigate human-introduced biases and inconsistencies [22]. OL uses methods from a diverse spectrum of fields such as machine learning, knowledge acquisition, natural-language processing, information retrieval, artificial intelligence and database management [23]. It is needed to emphasize that the insertion of statistics in ontologies leaded to the formation of the probabilistic ontology concept, which embodies the enabling of ontologies to represent uncertainty knowledge. Such uncertainty is present in knowledge proportionally to its complexity. How much complex the knowledge is how much uncertain it is.

Currently, it is widely accepted that systems that possess knowledge and are capable of decision making and reasoning are regarded as 'intelligent' [24]. There are recognised techniques, such as fuzzy logic, artificial neural networks, machine learning and evolutionary algorithms that contribute to increase a system's 'machine intelligence quotient' [25]. The rationale behind the intelligent label of those techniques is their ability to represent and deal with knowledge [26]. Consequently, in this paper, Artificial Neural Networks (ANN), Fuzzy Logic (FL) are addressed.

An ANN is an information-processing paradigm that is inspired by the way biological nervous system, such as brain, process information. Neural networks can be useful learning from existing data even when humans find it difficult to identify rules. Such as humans, ANN learn from experience and are able to adapt the Knowledge Base when facing new data. Focus on Ontology Learning, one application 
to consider is [27] where is proposed a method consisting of Projective Adaptive Resonance Theory neural network and Bayesian network probability theorem to automatically construct ontology. One problem related to ANN is if the neural network is implemented as a "black box", then any information "learned" by the network during this training is unavailable. Previous researchers, such as [28, 29] developed design techniques that allow network operation to be decoded after training. This researches made possible the automatic learning and adaptability of ANN with user's feedback related to the information learned.

In order to lead with uncertainty knowledge one solution is the application of Fuzzy Logic in OL. Fuzzy Logic is a multivalued logic able to absorb vague information, usually described in natural language, and convert it into a numerical format for easy computational manipulation, searching for shaping or emulate the human reasoning. In [30] is presented a fuzzy temporal model integrated with an ontology model to allow annotating ontology definitions with time specifications. Another successful application of FL to emulate human behaviour is the application of Fuzzy Logic to measure knowledge sharing, namely the confidence and knowledge complexity level [31].

In this sense, is possible to conclude that the referred learning technologies should be considered to implement a robust and complete intelligent system able to maintain by itself its knowledge. These may conduct authors to the possibility of build an (inspired human) prototype where semantics are provided by domain experts but which could be updated (maintained) by external users in a similar way as humans do when they learn through the others.

$\mathrm{KMa}$ is then proposed to be ruled by the analysis of the user's interactions feedback through OL. OL will facilitate the learning from the users usability in order to constantly improve the semantic interoperability between systems. This is accomplished by the insertion of statistics in ontologies leading to the formation of the probabilistic ontology concept, which embodies the enabling of ontologies to represent uncertainty knowledge.

\section{Conclusions}

The authors' research intended to contribute with a framework that allows the combination of semantic mappings with user's feedback in order to provide an automatic learning capability to ontologies enabling auto-adaptability of the information systems in the advent of dynamics. Such methodologies should be able to: 1) facilitate knowledge acquisition and maintenance increasing the intelligence of ontology based systems 2) work as a semantics mediator between enterprises communications with capacity to adapt to changes dynamics in the enterprises internal models 3) Improve the interoperability of software applications and information systems semantics.

Together all the mentioned methodologies are expected to contribute with ideas/solutions to the system's intelligence increase, which will facilitate semantics adaptability of systems.

In the scope do the research is intended to implement some prototype in order to prove or disprove the hypothesis. This will allow gathering of data and execution of 
tests according to some pre-established validation methods. Remarks concerning the implementation must be educe, since the research may find evidence that the prototype needs rectifications, the hypothesis failed the test or if it is necessary to reformulate the research questions (see Fig 1). Apart of having a set of main research questions related to the thesis research goal, there will be several sets of "small" research questions. For each of these questions is intended a publications about a specific topic, and sometimes a technology transfer to industry.

\section{References}

1. Gronau, N., Andresen, K.: Design and Use Patterns of Adaptability in Enterprise Systems. Reihe Wirtschaftsinformatik, Gito (2005)

2. Giusto, D., Iera, A., Morabito, G., Atzori, L.: The Internet of Things. Springer

3. Russomanno, D.J., Kothari, C., Thomas, O.: Sensor ontologies: from shallow to deep models. In: SSST, pp. 107-112 (2005)

4. W3C, W3c ssn incubator group report (2012), http: //www.w3 . org/2005/ Incubator/ssn/wiki/Incubator_Report

5. Venkataram, P.: An article to clear up some misconceptions about the nature of research (2012), Retrieved from the web: http: / cce.iisc.ernet.in/ motivationprinciples.pdf

6. Amaratunga, R., Baldry, D., Sarshar, M., Newton, R.: Qualitative and quantitative research in the built environment: application of "mixed" research approach: a conceptual framework to measure fm performance. Work Study (renamed International Journal of Productivity and Performance Management) 20, 17-31 (2002)

7. Camarinha-Matos, L.: Unit 2: Scientific method. In slides of the Scientific Research Methodologies and Techniques course of the PhD Program in Electrical and Computer Engineering of the FCT-UNL (2012), Retrieved from the web: http://www. uninova.pt/ cam/teaching/SRMT/SRMTunit2.pdf

8. Harris, D.: Creating a knowledge centric information technology environment. Harris Training \& Consulting Services Inc., Seattle (1996)

9. Carneiro, A.: How does knowledge management influence innovation and competitiveness? Journal of Knowledge Management, 87-98 (2000)

10. Leonard-Barton, D., Leonard, D.: Wellsprings of Knowledge: Building and Sustaining the Sources of Innovation. Harvard Business Press (1998)

11. Matthews, K., Harris, H.: Maintaining knowledge assets. In: Mathew, J., Kennedy, J., Ma, L., Tan, A., Anderson, D. (eds.) Engineering Asset Management, pp. 618-626. Springer, London (2006)

12. Metaxiotis, K.S., Ergazakis, K., Psarras, J.E.: Exploring the world of knowledge management: agreements and disagreements in the academic/practitioner community. J. Knowledge Management 9(2), 6-18 (2005)

13. Gruber, T.R.: A translation approach to portable ontology specifications. Knowl. Acquis. 5, 199-220 (1993)

14. An, Y.J., Chuan Huang, K., Geller, J.: Naturalness of ontology concepts for rating aspects of the semantic web (2006)

15. Sarraipa, J., Jardim-Gonçalves, R.: Semantics adaptability for systems interoperability (2010) 
16. Djedidi, R., Aufaure, M.-A.: Ontological knowledge maintenance methodology. In: Lovrek, I., Howlett, R.J., Jain, L.C. (eds.) KES 2008, Part I. LNCS (LNAI), vol. 5177, pp. 557-564. Springer, Heidelberg (2008)

17. Sarraipa, J.: Semantic adaptability for the systems interoperability. $\mathrm{PhD}$ thesis presented at Faculdade de Ciências e Tecnologia da Universidade Nova de Lisboa (2012)

18. Rolls, E.: Memory systems in the brain. Annual Review of Psychology (2000)

19. Patterson, K., Nestor, P.J., Rogers, T.T.: Where do you know what you know? The representation of semantic knowledge in the human brain. Nat. Rev. Neurosci. 8, 976-987 (2007)

20. Shamsfard, M., Abdollahzadeh Barforoush, A.: The state of the art in ontology learning: a framework for comparison. Knowl. Eng. Rev. 18, 293-316 (2003)

21. Gómez-Pérez, A., Manzano-Macho, D.: A survey of ontology learning methods and techniques. Deliverable 1.5, OntoWeb Consortium (2003)

22. Zhou, L.: Ontology learning: state of the art and open issues. Inf. Technol. and Management 8 , 241-252 (2007)

23. Sabou, M.: Learning domain ontologies for web service descriptions: An experiment in bioinformatics. In: Intl. World Wide Web Conf., WWW (2005)

24. Meystel, A.M., Albus, J.S.: Intelligent Systems: Architecture, Design, and Control, 1st edn. John Wiley \& Sons, Inc., New York (2000)

25. Zadeh, L.A.: Fuzzy logic, neural networks, and soft computing. Commun. ACM 37, 77-84 (1994)

26. Kasabov, N., Filev, D.: Evolving intelligent systems: methods, learning, \& applications. Evolving Fuzzy Systems (2006)

27. Xi-Hu, Z., Yan-Fei, L.: Building ontology automatically based on bayesian network and part neural network. In: Proceedings of the 2009 WRI Global Congress on Intelligent Systems, GCIS 2009, vol. 04, pp. 563-566. IEEE Computer Society, Washington, DC (2009)

28. Fu, L.: Rule generation from neural networks. Systems, Man and Cybernetics (1994)

29. Towell, G.G., Shavlik, J.W.: The extraction of refined rules from knowledge-based neural networks. Machine Learning, 71-101 (1993)

30. Nagypál, G., Motik, B.: A fuzzy model for representing uncertain, subjective and vague temporal knowledge in ontologies. In: Meersman, R., Tari, Z., Schmidt, D.C. (eds.) CoopIS/DOA/ODBASE 2003. LNCS, vol. 2888, pp. 906-923. Springer, Heidelberg (2003)

31. Zadjabbari, B., Mohseni, S., Wongthongtham, P.: Fuzzy logic based model to measure knowledge sharing. In: 3rd IEEE International Conference on Digital Ecosystems and Technologies (2009) 